\title{
Increased histone deacetylase 6 expression serves as a favorable prognostic factor for diffuse large B-cell lymphoma
}

\author{
Xiao-Ji Lin' \\ Li-Meng Cai ${ }^{2}$ \\ Zi-Jun Qian ${ }^{2}$ \\ Chen-Yi Wang ${ }^{2}$ \\ Ni Sun' \\ Xiao-Hai Sun' \\ He Huang' \\ Wen-Jian Guo' \\ Hai-Yan Lin' \\ Rong-Xin Yao' \\ 'Department of Haematology, The \\ Second Affiliated Hospital and Yuying \\ Children's Hospital of Wenzhou \\ Medical University, ${ }^{2}$ Science and \\ Technology of Students Association, \\ The Second School of Medicine, \\ Wenzhou Medical University, \\ Wenzhou, Zhejiang, People's \\ Republic of China
}

\author{
This article was published in the following Dove Press journal: \\ OncoTargets and Therapy \\ 24 October 2017 \\ Number of times this article has been viewed
}

Objective: This study aims to investigate ectopic expression of histone deacetylase 6 (HDAC6) in diffuse large B-cell lymphoma (DLBCL).

Methods: This study analyzed patients with DLBCL ( $\mathrm{n}=132)$ and reactive lymph node hyperplasia $(n=32)$ diagnosed in our hospital from December 2007 to May 2016. Correlation between HDAC6 expression and clinical pathologic features was analyzed by $\chi^{2}$ test. The significant differences between the 5-year overall survival (OS) or progression-free survival (PFS) and high HDAC6 expression as well as DLBCL clinic-pathological features including age, International Prognostic Index (IPI) score, Eastern Cooperative Oncology Group score, lactate dehydrogenase (LDH), and germinal center B-cell-like were assessed by univariate and multivariate analyses.

Results: HDAC6 high-expression percentage in DLBCL was significantly higher than that in the control group. The proportion of IPI score of $0-2,5$-year OS, and PFS in the high-expression group, which had lower percentage of patients with increased LDH and $\beta_{2}$-microglobulin, were significantly higher than those in the low-expression group. Moreover, HDAC6 mRNA expression in HDAC6 protein low expression was markedly lower than that in protein high expression. The multivariate analysis demonstrated that HDAC6 high expression was an independent prognostic factor for patients with DLBCL.

Conclusion: HDAC6 high expression might be a prognostic factor for DLBCL.

Keywords: diffuse large B-cell lymphoma, HDAC6, prognosis

\section{Introduction}

Diffuse large B-cell lymphoma (DLBCL) is the most common type of non-Hodgkin's lymphoma (NHL) according to the Revised European-American Classification of Lymphoid Neoplasms and the World Health Organization classification criteria for malignant lymphoma. DLBCL is a group of very heterogeneous B-cell lymphoma and accounts for $30 \%-40 \%$ of NHL. ${ }^{1}$ Clinical course of DLBCL was invasive. Although the long-term remission rate of the disease has been further improved with the introduction of rituximab, there are some patients with poor efficacy of DLBCL. Therefore, domestic and foreign scholars are keen to study the pathogenesis and prognostic factors for DLBCL.

It is well known that histone modification plays an important role in regulating gene expression and is also recognized as one of the most important mechanisms for the development of tumor. In the process of histone modification, histone deacetylase (HDAC) regulates the deacetylation of nucleosome histones and enhances the interaction between histones and DNA, thereby leading to transcriptional silencing. Thus, the abnormal binding to a particular promoter region of normal functional genes associated with the regulation of tumor proliferation, migration, angiogenesis, and so
Correspondence: Hai-Yan Lin Department of Haematology, The Second Affiliated Hospital and Yuying Children's Hospital of Wenzhou Medical University, 109 Xueyuan West Road, Lucheng District, Wenzhou 325000, Zhejiang, People's Republic of China Email haiyan_lin234@163.com 
on, by HDAC could cause genes' transcriptional inhibition consequently resulting in malignancy. ${ }^{2,3}$ HDAC6 is a special type of HDAC family and locates on the Xp11.23 chromosome, which mainly expressed in the heart, liver, kidney, testis, brain, and pancreas. Current studies have shown that HDAC6 is a necessary gene for the process of tumor transformation and formation and can be induced in the process of proto-oncogene Ras. ${ }^{4}$ Zhang et $\mathrm{al}^{5}$ found that HDAC6 expression in invasive breast cancer was closely related to tumor size, low histological grade, and estrogen receptor and progesterone receptor positive, and also high expression of HDAC6 is a good prognostic factor for patients.

The study also confirmed that there was ectopic expression of HDAC6 in DLBCL. Cai et $\mathrm{al}^{6}$ used Western blotting to study the expression of HDAC6 in three DLBCL cell lines and found that three cell lines showed different levels of HDAC6 expression and Trichostatin A (HDAC inhibitor) significantly inhibited the proliferation of these cell lines. However, there is little research on the role and clinical significance of ectopic expression of HDAC6 in DLBCL. Herein, we aim to explore the expression and clinical significance of HDAC6 in DLBCL and provide reliable predictors for the prognosis of DLBCL.

\section{Methods}

\section{General data for objects}

We performed retrospective analysis on 132 DLBCL patients who diagnosed in our hospital from December 2007 to May 2016, with 32 cases of reactive lymph node hyperplasia patients as control. The general data for DLBCL patients are as follows: median age 57.6 (16-78) years; 75 males and 57 females; and 75 cases of primary extranodular lymph nodes and 57 cases of primary intranodal lymph nodes (including 27 cases of gastrointestinal tract, six cases of mediastinal, four cases of testis/ovary, two cases of central nervous system, seven cases of liver/spleen, three cases of nasopharyngeal, two cases of thyroid, and five cases of other parts). The follow-up time was death or November 10, 2016, with a median follow-up time of 49 (5-102) months and a loss to follow-up rate of $7.5 \%$. All samples were obtained after receiving written informed consent forms from all patients for this study, and the experimental protocols were approved by Human Ethics Committee of The Second Affiliated Hospital and Yuying Children's Hospital of Wenzhou Medical University.

\section{Immunohistochemistry}

All fresh samples were fixed in 4\% formaldehyde and, then, embedded in paraffin followed by made into $3-5 \mu \mathrm{m}$ thickness sections for immunohistochemically staining (streptavidin/peroxidase method). In accordance with the manufacturers' instructions, the identified positive tissue slices were used as a positive control and phosphate buffered saline instead of primary antibody was used as a negative control. The expression of HDAC6 was detected by HDAC6 mouse antihuman monoclonal antibody (Abcam, Cambridge, UK) and positive for cytoplasmic brown granules. Staining degree score standard is as follows: no coloring for 0 points, light coloring for 1 point, moderate coloring for 2 points, and depth coloring for 3 points. Positive cell percentage score standard is as follows: $\leq 5 \%$ for 0 point, $6 \%-25 \%$ for 1 point, $26 \%-50 \%$ for 2 points, and $\geq 51 \%$ for 3 points. Total scores derive from product of above scores are as follows: $0-1$ for negative (-), 2-3 for weak positive $(+), 4-6$ for medium positive $(++)$, and $>6$ for strong positive $(+++)$. According to the total scores, HDAC6 expression was divided into low-expression group (-/+) and high-expression group (++ to +++$)$ as described in Zhang et al. ${ }^{5}$

\section{Quantitative real-time polymerase chain reaction}

Thirty-five lymphoma tissue samples collected from DLBCL patients and 15 hyperplasia lymph node samples isolated from reactive lymph node hyperplasia patients from 2013 to 2016 were frozen at $-80^{\circ} \mathrm{C}$ with liquid nitrogen for quantitative real-time PCR and partly were fixed with $4 \%$ formaldehyde for immunohistochemistry. For quantitative real-time PCR, tissues were crushed under liquid nitrogen and total RNA from tissue were extracted by the TRIzol reagent. Concentration and purity of the extracted samples were analyzed using an ultraviolet spectrophotometer. Reverse transcription was performed using ReverTran Ace qPCR RT Kit (ToYoBo, Osaka, Japan). All operations were performed on ice, and the total reaction system was $10 \mu \mathrm{L}$. The reverse transcription reaction product was used as a template for amplification reaction. The primers used for PCR were designed by Primer 5.0 software. Primers for HDAC6 are as follows: upstream primer 5'-TCAGGTCTACTGTGGTCGTT-3' and downstream 5'-TCTTCACATCTAGGAGAGCC-3'. The glyceraldehyde-phosphate dehydrogenase (GAPDH) upstream primer is 5'-AAATCCCATCACCATCTTCC-3', and the GAPDH downstream primer is $5^{\prime}$-GCTCAG TGTAGCCCAGGAT-3'. The reagents used for PCR were $10 \mu \mathrm{L}$ SYBR Premix Ex Taq ${ }^{\mathrm{TM}}$ II, $0.8 \mu \mathrm{L}$ forward primer, $0.8 \mu \mathrm{L}$ reverse primer, $0.4 \mu \mathrm{L}$ ROX Reference Dye, and $8 \mu \mathrm{L}$ cDNA. Amplification reaction was placed on the ABI7300 fluorescence quantitative PCR instrument according to the following conditions: predenaturation at $95^{\circ} \mathrm{C}$ for $30 \mathrm{~s}$ and 
40 cycles of amplification at $95^{\circ} \mathrm{C} 5 \mathrm{~s}$ and $60^{\circ} \mathrm{C} 31 \mathrm{~s}$. After the PCR, DHAC6 mRNA expression was analyzed by the ABI 7300 System SDS Software. After adjusting the baseline cycle and calculating the value of the baseline, the expression level of the gene to be tested was calculated according to the standard curve. The ratio of the gene expression/ housekeeping gene GAPDH was statistically analyzed.

\section{Therapy}

All patients with DLBCL received cyclophosphamide, doxorubicine, vincristine, prednisone (CHOP) or rituximab plus CHOP (R-CHOP) regimen chemotherapy. The treatment course is 3 weeks, and the median chemotherapy course is 5 (4-8). A range of radiotherapy (range 8-50 Gy, median $45 \mathrm{~Gy}$ ) was given to patients with residual lesions or a large tumor before chemotherapy.

\section{Statistical analysis}

All data are analyzed by using SPSS13.0 statistical software. The relationships between HDAC6 expression and different clinical features as well as pathological subtypes were performed by $\chi^{2}$ test. The paired $t$-test with two tail was used to compare measurement data of the two groups. The study endpoints were the overall survival (OS) and progressionfree survival (PFS). Survival analysis was performed using Kaplan-Meier analysis and log-rank test. Univariate and multivariate analyses using Cox regression were used to assess the significance of differences between the 5-year OS or PFS and high HDAC6 expression as well as DLBCL clinic-pathological features including age, International Prognostic Index (IPI) score, Eastern Cooperative Oncology Group (ECOG) score, lactate dehydrogenase (LDH), and germinal center B-cell-like (GCB). OS is defined as the initial diagnosis to death or last follow-up time, and PFS is defined as the effective onset of treatment to lymphoma progression, recurrence, death, or last follow-up time. $P<0.05$ was considered statistically significant.

\section{Results \\ HDAC6 protein expression in DLBCL patients}

In lymphoma tissue of DLBCL patients, HDAC6 was brown granules and mainly located in the cytoplasm of cells (Figure 1). Among 132 cases of DLBCL patients, lowexpression group contained 39 cases (29.5\%), which included 17 cases in weak positive staining and 22 cases in negative staining; high-expression group had 93 cases (70.5\%), which included 34 cases of moderate positive staining and 59 cases of strong positive. The cases (\%) of high-expression group and low-expression group in 32 cases of reactive lymph node hyperplasia patients were 7 cases $(21.9 \%)$ and 25 cases (78.1\%), respectively. There were significant differences in expression level of HDAC6 between two types of patients $\left(\chi^{2}=25.54, P<0.001\right)$ (Table 1).

\section{HDAC6 mRNA expression in DLBCL patients}

The relative expression level of HDAC6 mRNA in 35 cases of DLBCL patients was 7.2271 \pm 3.39369 , and that in 15 cases of lymph node reactive hyperplasia patients was $1.8835 \pm 0.91433$. The expression of HDAC6 mRNA in DLBCL was significantly higher than that in lymph node hyperplasia $(t=-6.352, P<0.001)$ (Figure 2). Furthermore, as shown in Figure 3, 10 DLBCL patients with HDAC6 protein low expression and 25 patients with HDAC6 protein high expression were observed according to immunohistochemistry. The mRNA expression of HDAC6 protein low expression (3.1740 \pm 1.61934$)$ was markedly lower than that in protein high expression (8.8492 \pm 2.39726$)$, and the difference was extremely significant $(t=-6.856, P<0.001)$.

\section{Correlation of high expression of HDAC6 to clinical features}

As shown in Table 2, the proportion of patients with 3-5 IPI score in the HDAC6 high-expression group was significantly
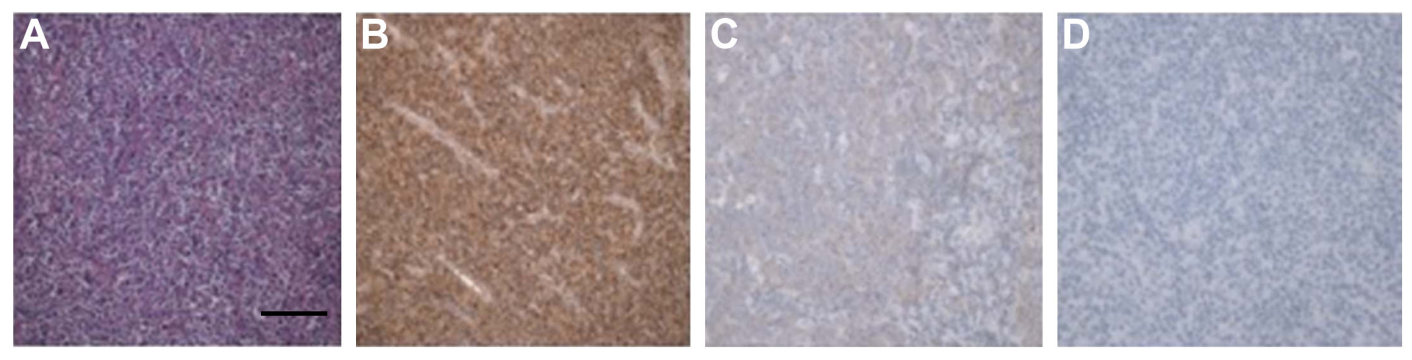

Figure I Immunohistochemistry detects HDAC6 in DLBCL patient.

Notes: Scale bar $50 \mu \mathrm{m}$. Magnification is $\times 200$. (A) Centroblasts were distributed in a diffuse way. (B) Most tumors displayed strong positive expression. (C) Most tumors displayed weak positive expression. (D) Most tumors displayed negative expression.

Abbreviations: DLBCL, diffuse large B-cell lymphoma; HDAC6, histone deacetylase 6. 
Table I HDAC6 expression in DLBCL patients and reactive lymph node hyperplasia patients, cases (\%)

\begin{tabular}{lllll}
\hline Patients & $\begin{array}{l}\text { Low- } \\
\text { expression } \\
\text { group }\end{array}$ & $\begin{array}{l}\text { High- } \\
\text { expression } \\
\text { group }\end{array}$ & $\chi^{2}$ value & P-value \\
\hline DLBCL & $39(29.5)$ & $93(70.5)$ & 25.54 & 0.001 \\
Reactive lymph & $25(78.1)$ & $7(21.9)$ & & \\
node hyperplasia & & & & \\
\hline
\end{tabular}

Abbreviations: DLBCL, diffuse large B-cell lymphoma; HDAC6, histone deacetylase 6 .

lower than that in the HDAC6 low-expression group (28\%, $26 / 93$ vs $51.3 \%, 20 / 39)(P=0.01)$. The proportion of patients with increased LDH in the high-expression group was significantly lower than that in the low-expression group $(32.3 \%, 30 / 93$ vs $53.8 \%, 21 / 39)(P=0.02)$; meanwhile, the proportion of patients with increased $\beta_{2}$-microglobulin in the high-expression group was also lower than that in the lowexpression group $(31.2 \%, 29 / 93$ vs $56.4 \%, 22 / 39)(P<0.05)$. There was no significant difference in sex, age, Ann Arbor stage, ECOG score, GCB type, B symptom, and treatment regimen between low-expression group and high-expression group $(P>0.05)$.

\section{High expression of HDAC6 positively correlated to patients' survival}

The 5-year OS (41.9\%) of HDAC6 low-expression group was significantly lower than that of HDAC6 high-expression group $(69.0 \%)\left(\chi^{2}=10.23, P=0.001\right)$ (Figure $\left.4 \mathrm{~A}\right)$. The 5 -year PFS of the low- and high-expression groups was 34.0 and $54.4 \%$, respectively, and had significant difference between

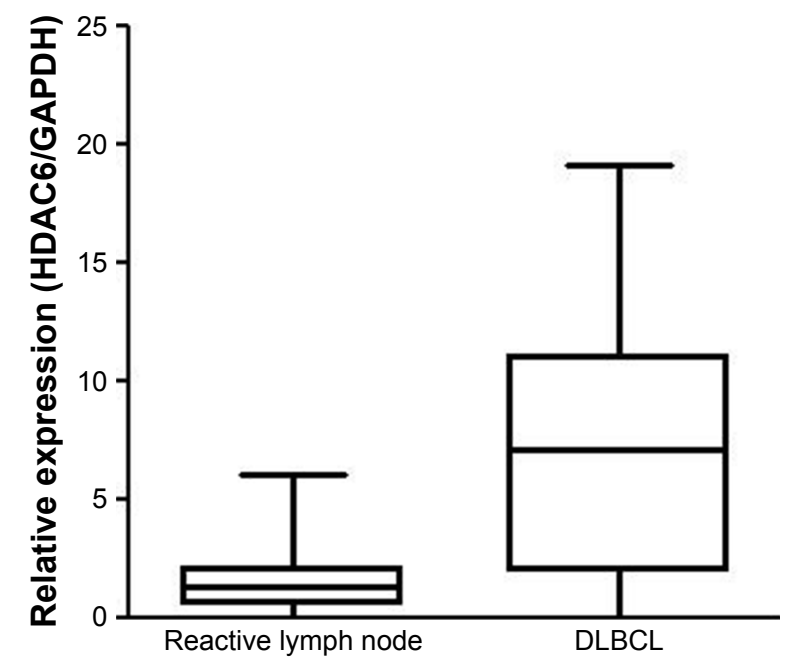

Figure 2 Quantitative real-time PCR detects HDAC6 mRNA expression. Abbreviations: PCR, polymerase chain reaction; DLBCL, diffuse large B-cell lymphoma; HDAC6, histone deacetylase 6.

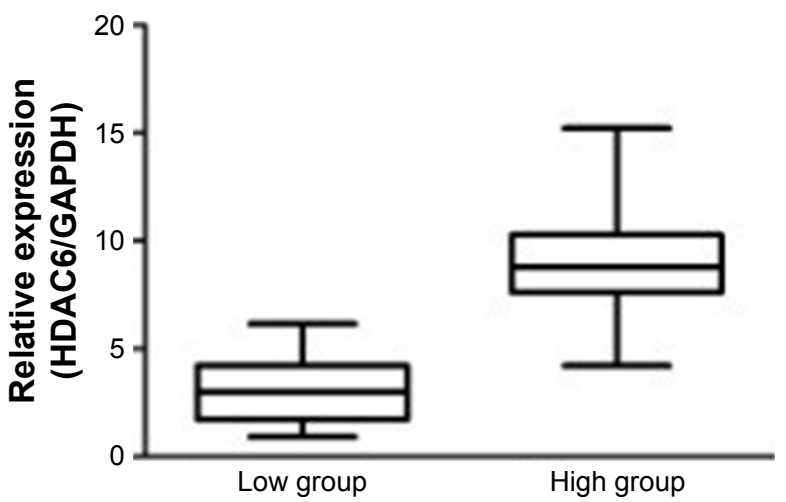

Figure 3 HDAC6 mRNA expression in HDAC6 low- and high-expression groups. Abbreviation: HDAC6, histone deacetylase 6.

Table 2 Correlation between HDAC6 expression and DLBCL clinic-pathological features, cases (\%)

\begin{tabular}{|c|c|c|c|c|}
\hline Clinical features & $\begin{array}{l}\text { Low- } \\
\text { expression } \\
\text { group }(n=39)\end{array}$ & $\begin{array}{l}\text { High- } \\
\text { expression } \\
\text { group }(n=93)\end{array}$ & $\chi^{2}$ value & $P$-value \\
\hline Sex & & & 0.004 & 0.951 \\
\hline Male & $22(56.4)$ & $53(57)$ & & \\
\hline Female & $17(43.6)$ & $40(43)$ & & \\
\hline Age (years) & & & 0.318 & 0.573 \\
\hline$\leq 60$ & $24(6 \mid .5)$ & $62(66.7)$ & & \\
\hline$>60$ & $15(38.5)$ & 31 (33.3) & & \\
\hline Ann Arbor stage & & & 0.63 & 0.427 \\
\hline $\mathrm{I} / \mathrm{II}$ & $2 \mathrm{I}(53.8)$ & $57(6 \mid .3)$ & & \\
\hline III/IV & 18 (46.2) & $36(38.7)$ & & \\
\hline IPI score & & & 6.584 & 0.01 \\
\hline $0-2$ scores & $19(48.7)$ & $67(72)$ & & \\
\hline $3-5$ scores & $20(5 \mid .3)$ & $26(28)$ & & \\
\hline ECOG score & & & 0.007 & 0.933 \\
\hline$<2$ scores & 35 (89.7) & $83(89.2)$ & & \\
\hline$\geq 2$ scores & $4(10.3)$ & $10(10.8)$ & & \\
\hline$\beta_{2}$-microglobulin & & & 7.376 & 0.007 \\
\hline Increase & $22(56.4)$ & $29(31.2)$ & & \\
\hline Normal & $17(43.6)$ & $64(68.8)$ & & \\
\hline $\mathrm{LDH}$ & & & $5.40 \mathrm{I}$ & 0.02 \\
\hline Increase & $21(53.8)$ & $30(32.3)$ & & \\
\hline Normal & 18 (46.2) & $63(67.7)$ & & \\
\hline B symptom & & & 2.114 & 0.146 \\
\hline Yes & I5 (38.5) & $24(25.8)$ & & \\
\hline None & $24(61.5)$ & $69(74.2)$ & & \\
\hline Immunophenotype & & & 0.486 & 0.486 \\
\hline GCB & $13(33.3)$ & 37 (39.8) & & \\
\hline Non-GCB & $26(66.7)$ & $56(60.2)$ & & \\
\hline Location & & & 0.004 & 0.951 \\
\hline $\begin{array}{l}\text { Inside primary } \\
\text { node }\end{array}$ & $22(56.4)$ & $53(57)$ & & \\
\hline $\begin{array}{l}\text { Outside primary } \\
\text { node }\end{array}$ & $17(43.6)$ & $40(43)$ & & \\
\hline Therapy & & & 0.092 & 0.761 \\
\hline $\mathrm{CHOP}$ & $25(64.1)$ & $57(6 \mid .3)$ & & \\
\hline $\mathrm{R}-\mathrm{CHOP}$ & $14(35.9)$ & $36(38.7)$ & & \\
\hline
\end{tabular}

Abbreviations: DLBCL, diffuse large B-cell lymphoma; HDAC6, histone deacetylase 6; IPI, International Prognostic Index; ECOG, Eastern Cooperative Oncology Group; LDH, lactate dehydrogenase; GCB, germinal center B-celllike; CHOP, cyclophosphamide, doxorubicine, vincristine, prednisone; R-CHOP, rituximab plus $\mathrm{CHOP}$. 

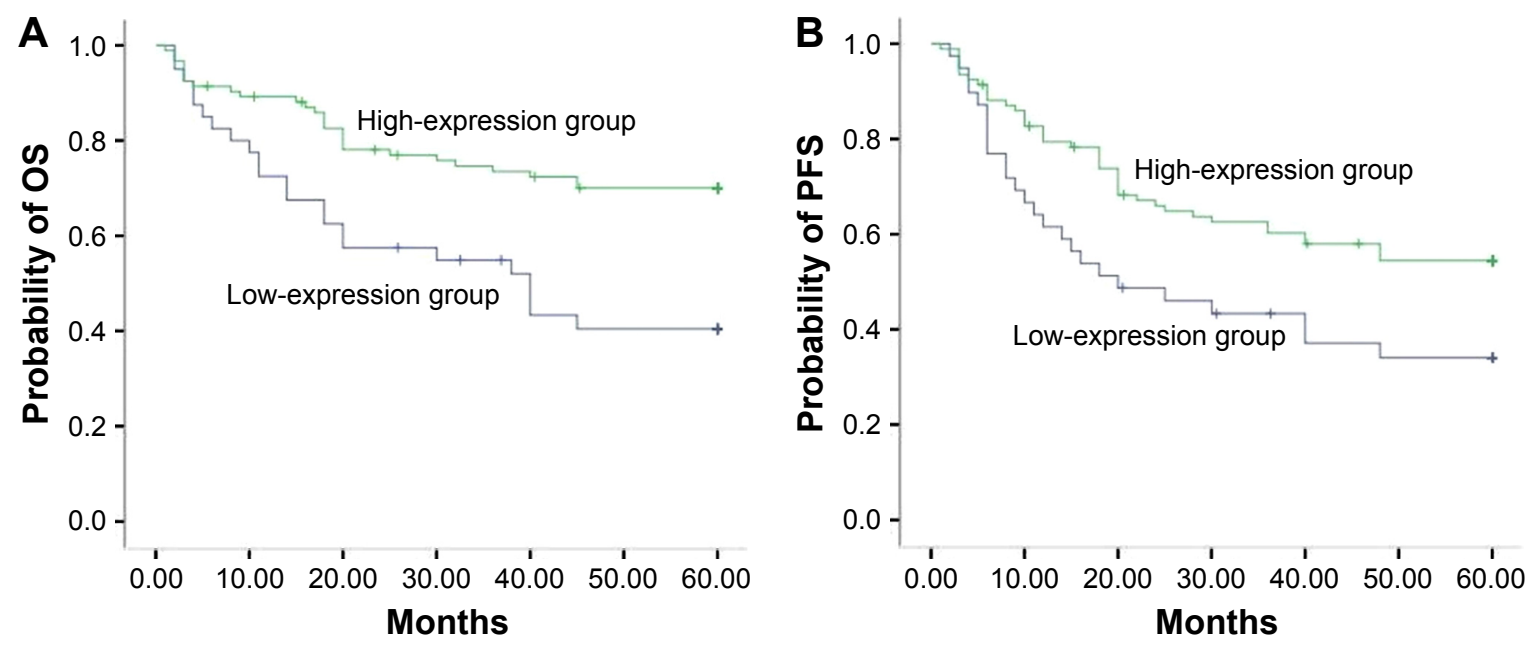

Figure 4 Correlation of HDAC6 expression with patients' survival.

Notes: (A) OS. (B) PFS.

Abbreviations: $\mathrm{HDAC6}$, histone deacetylase 6; OS, overall survival; PFS, progression-free survival.

these groups $\left(\chi^{2}=5.80, P=0.016\right)$ (Figure 4B). As shown in Figure 5, the 5-year OS in low- and high-expression groups was 37.6 and $63.1 \%$, respectively, with no significant differences $\left(\chi^{2}=2.504, P=0.114\right)$ (Figure 5A), while significant differences of the 5-year PFS were observed in low- $(32.5 \%)$ and high-expression (49.4\%) groups $\left(\chi^{2}=4.690, P=0.030\right)$ (Figure 5B) when DLBCL patients received CHOP regimen chemotherapy. On the contrary, R-CHOP regimen led to 49.0 and $80.5 \%$, respectively, of 5-year OS in low-expression and high-expression groups $\left(\chi^{2}=5.117, P=0.024\right)$ (Figure 6A); however, the 5-year PFS in aforementioned two groups was 40.8 and $77.8 \%$, respectively $\left(\chi^{2}=6.016, P=0.014\right)$ (Figure 6B).

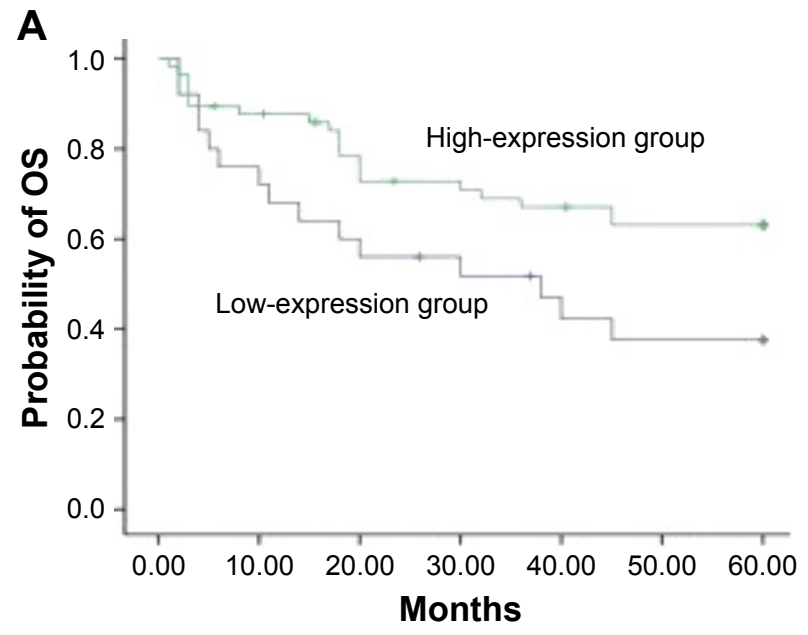

\section{Analysis of prognostic factors of DLBCL patients}

As shown in Tables 3 and 4, univariate analysis revealed that high HDAC6 expression, age ( $\leq 60$ years), Ann Arbor staging, ECOG score $(<2)$, increased LDH, and GCB observably affected 5-year OS, while high HDAC6 expression, age ( $\leq 60$ years), Ann Arbor staging, ECOG score $(<2)$, and GCB were the independent prognostic factors for 5-year OS of patients with DLBCL based on multivariate analysis. Similarly, high HDAC6 expression, age ( $\leq 60$ years), Ann Arbor staging, R-CHOP, and ECOG score $(<2)$ were the independent prognostic factors for 5-year PFS of patients with DLBCL according to univariate and multivariate analyses.

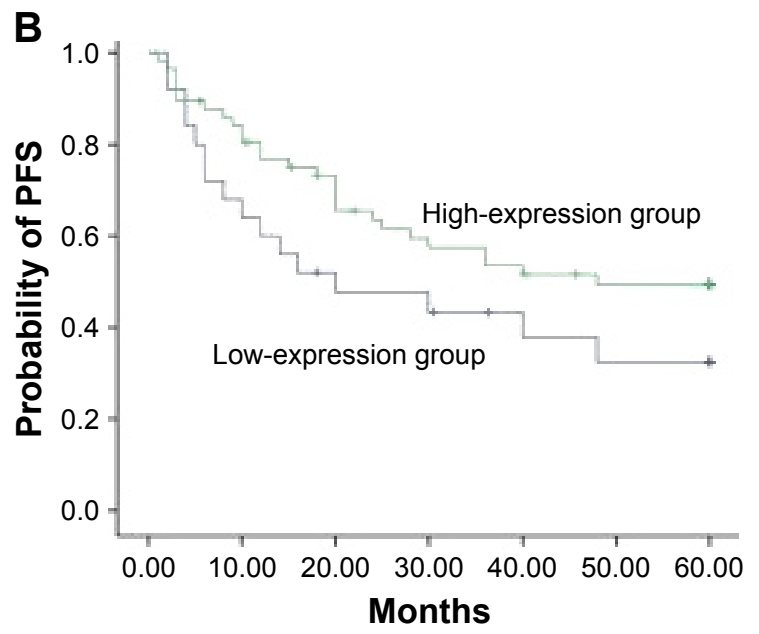

Figure 5 Correlation of HDAC6 expression with patients' survival receiving CHOP regimen. 

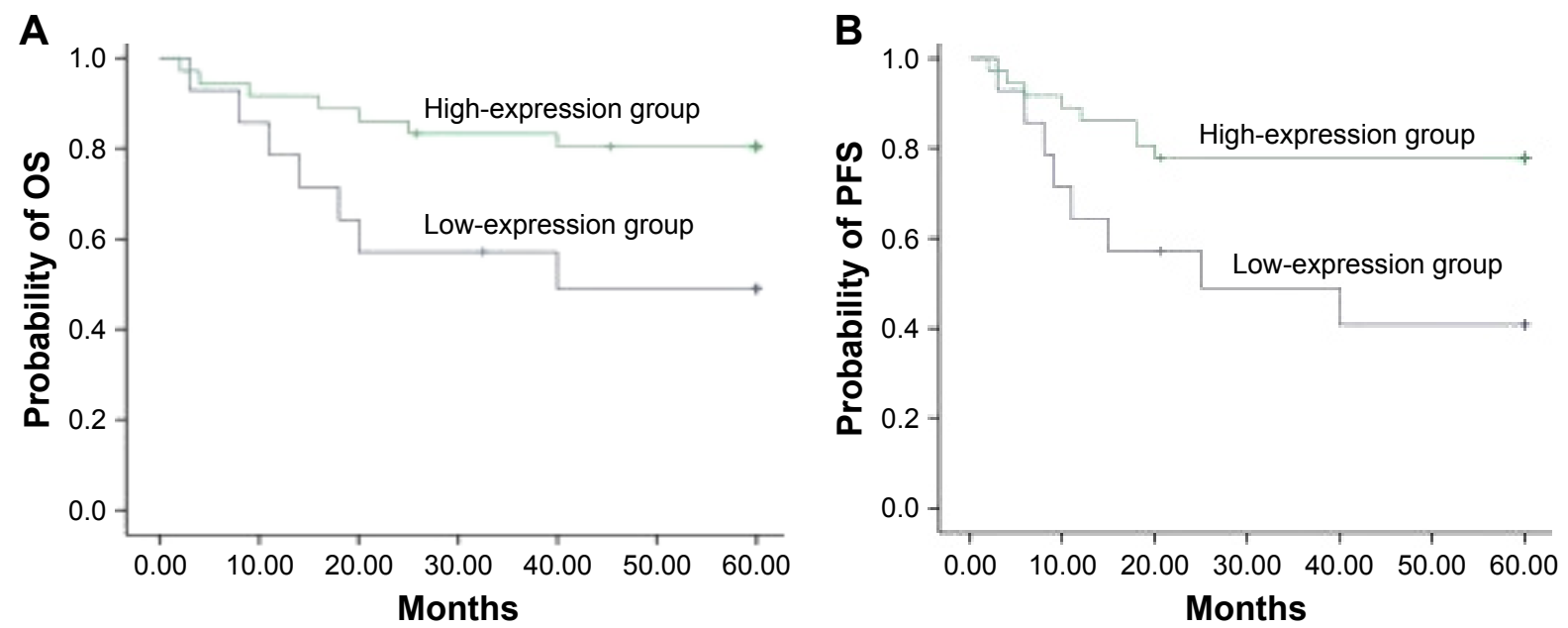

Figure 6 Correlation of HDAC6 expression with patients' survival receiving R-CHOP regimen.

Notes: (A) OS. (B) PFS

Abbreviations: HDAC6, histone deacetylase 6; OS, overall survival; PFS, progression-free survival; CHOP, cyclophosphamide, doxorubicine, vincristine, prednisone; $\mathrm{R}-\mathrm{CHOP}$, rituximab plus $\mathrm{CHOP}$.

\section{Discussion}

As is known to us that the IPI is the most classic prognostic system for DLBCL patients, but IPI does not address the molecular biology of tumors, even though the prognosis of patients with the same IPI score may still be quite different. ${ }^{7}$ So domestic and foreign scholars have been committed to find the prognosis-associated markers for DLBCL. Our present study recognized HDAC6 high expression as favorable prognostic event.

Acetylation and deacetylation of histones are key steps in the process of DNA replication, transcription, and repair and cocontrolled by HDAC and histone acetyltransferase. ${ }^{8}$ In the state of cell transformation, HDAC activity was significantly enhanced and thereby made the original gene expression balance broke, leading to transcriptional inhibition of a variety of normal functional gene that contributes to malignant tumor. ${ }^{9}$ On count of this, the application of HDAC inhibitor has been promisingly accepted for targeting anticancer drugs and has

Table 3 Prognostic factors analysis of 5-year OS

\begin{tabular}{|c|c|c|c|}
\hline \multirow[t]{2}{*}{ Parameter } & \multirow{2}{*}{$\frac{\text { Univariate }}{P \text {-value }}$} & \multicolumn{2}{|c|}{ Multivariate } \\
\hline & & $P$-value & $\operatorname{RR}(95 \% \mathrm{Cl})$ \\
\hline Age ( $\leq 60$ years $)$ & 0.001 & $<0.001$ & $3.256(1.813-5.846)$ \\
\hline Increased LDH & 0.014 & 0.169 & $0.670(0.379-I .185)$ \\
\hline ECOG scores $(<2)$ & 0.003 & 0.013 & $2.603(1.225-5.533)$ \\
\hline GCB & 0.024 & 0.016 & $2.239(1.165-4.304)$ \\
\hline HDAC6 high expression & 0.001 & 0.006 & $0.443(0.249-0.790)$ \\
\hline Ann Arbor staging & $<0.001$ & $<0.001$ & 3.321 (1.849-5.964) \\
\hline $\mathrm{R}-\mathrm{CHOP}$ & 0.053 & 0.082 & $0.566(0.298-1.074)$ \\
\hline
\end{tabular}

Abbreviations: HDAC6, histone deacetylase 6; OS, overall survival; ECOG, Eastern Cooperative Oncology Group; LDH, lactate dehydrogenase; GCB, germinal center B-cell-like; CHOP, cyclophosphamide, doxorubicine, vincristine, prednisone; $\mathrm{R}-\mathrm{CHOP}$, rituximab plus CHOP; RR, rate ratios. been shown to have a good therapeutic effect in a variety of malignancies, which mainly blocks tumor cell growth through the inhibition of HDAC activity. ${ }^{10-13}$

HDAC6 was type II HDACs, which was first cloned in two different research groups in Grozinger et a ${ }^{14}$ and Verdel and Khochbin. ${ }^{15}$ HDAC6 is a relatively unique HDAC that participates in cell migration, immune synaptic formation, lymphocyte chemotaxis, and intracellular error-folding protein degradation and stress responses. ${ }^{16,17}$ Studies also have shown that HDAC6 has very close relationship to tumor development. HDAC6 has been shown to be highly expressed in oral squamous cell carcinoma, breast cancer, lung cancer, and kidney cancer. ${ }^{18-20}$ Hideshima et $\mathrm{a}^{21}$ found that six of the seven different myeloma cell lines were highly expressed HDAC6, whereas only one showed low expression of HDAC6; further study of 6 cases of HDAC6-positive myeloma with Tubacin (specific inhibition HDAC6) treatment for $72 \mathrm{~h}$ showed that all cell lines were inhibited in different

Table 4 Prognostic factors analysis of 5-year PFS

\begin{tabular}{|c|c|c|c|}
\hline \multirow[t]{2}{*}{ Parameter } & \multirow{2}{*}{$\frac{\text { Univariate }}{P \text {-value }}$} & \multicolumn{2}{|c|}{ Multivariate } \\
\hline & & $P$-value & $\mathbf{R R}(95 \% \mathrm{CI})$ \\
\hline Age ( $\leq 60$ years) & $<0.001$ & $<0.001$ & $3.568(2.113-6.023)$ \\
\hline ECOG scores $(<2)$ & $<0.001$ & 0.001 & $3.088(1.615-5.904)$ \\
\hline HDAC6 high expression & 0.012 & 0.012 & $0.510(0.30 I-0.864)$ \\
\hline Ann Arbor staging & $<0.001$ & $<0.001$ & $3.456(2.012-5.938)$ \\
\hline $\mathrm{R}-\mathrm{CHOP}$ & 0.001 & 0.003 & $0.400(0.219-0.730)$ \\
\hline Increased LDH & 0.245 & & \\
\hline GCB & 0.057 & & \\
\hline
\end{tabular}

Abbreviations: HDAC6, histone deacetylase 6; PFS, progression-free survival; ECOG, Eastern Cooperative Oncology Group; LDH, lactate dehydrogenase; GCB, germinal center B-cell-like; CHOP, cyclophosphamide, doxorubicine, vincristine, prednisone; R-CHOP, rituximab plus $\mathrm{CHOP}$; RR, rate ratios. 
degrees. Zhang et $\mathrm{al}^{5}$ and Seo et $\mathrm{a}^{22}$ studied the expression of HDAC6 mRNA in relation to age, tumor size, low histological grade, HER2 gene amplification, and estrogen receptor- and progesterone receptor-positive tumors. Lee et $\mathrm{al}^{4}$ showed that knockdown of HDAC6 was 5-30 times inhibited the proliferation of SKOV3 transformed ovarian cancer cell line, SKBR3 breast cancer cell line, and MCF-7 breast cancer cell line; moreover, HDAC6-specific shRNA injected subcutaneously to SCID (severe combined immunodeficient) mice inhibited tumor growth and further reconstitution of HDAC6 knockout mice with wild-type HDAC6 can regain the initial phenotype, indicating that HDAC6 is necessary during tumor growth. These studies suggest that HDAC6 plays an important role in the development of tumors and has even become an important molecular target for the treatment of tumors. ${ }^{23}$

Phase II clinical study of Duvic et $\mathrm{al}^{24}$ and Olsen et $\mathrm{al}^{25}$ found that vorinostat (broad-spectrum HDAC inhibitor) achieved good clinical efficacy in the treatment of refractory skin T-cell lymphoma, making the drug approved by the FDA in 2006 for the treatment of refractory recurrent CTCL. Marquard et $\mathrm{al}^{26}$ demonstrated that HDAC6 was found to be abnormally expressed in skin T-cell lymphoma, that HDAC6 expression was positively correlated with prognosis, and that patients with high expression of HDAC6 had a longer survival time. This further confirms that the development of malignant lymphoma is closely related to the abnormality of HDAC6. However, little is known about the expression of HDAC6 in DLBCL and its correlation with clinic-pathological features and prognosis. Marquard et $\mathrm{al}^{27}$ showed that HDAC6 in 31 cases of DLBCL with abnormal expression and the expression rate as high as $71 \%$ and patients with HDAC6 high expression may have a good prognosis. The results of this study show that $69.9 \%$ of the 132 DLBCL patients showed high expression of HDAC6, which was consistent with the reported literature. The proportion of DLBCL patients with IPI 3-5, increased LDH, and increased $\beta_{2}$-microglobulin in HDAC6 high-expression group were lower than that in patients with HDAC6 low-expression group, suggesting that DLBCL patients were with ectopic HDAC6 expression and patients with HDAC6 high expression were with low IPI score and tumor load characteristics. Also, in this study, we found that the 5-year OS and PFS of HDAC6 overexpression group were significantly higher than those of low-expression group. This result highly suggests that HDAC6 expression may be a good prognostic factor for DLBCL patients. Accumulating evidence has evaluated the potential role of R-CHOP therapeutical regimen for patients with CD20 DLBCL. ${ }^{28}$ In this study, we performed stratified analysis for the effect of HDAC6 expression on patients' survival rates to eliminate the interference of therapeutic schemes in prognosis and the multivariate analysis suggested that increased HDAC6 expression was a suitable prognostic factor for patients with DLBCL.

\section{Conclusion}

HDAC6 was highly expressed in DLBCL patients, whereas HDAC6 high expression was a good prognostic factor for DLBCL patients. Thus, we can provide a more accurate prognostic judgment for DLBCL patients by analyzing HDAC6 expression. In the future study, we will focus on the role of HDAC6 in the development of DLBCL and also for the more effective treatment of DLBCL to provide theoretical basis and new ideas.

\section{Disclosure}

All authors report no conflicts of interest in this work.

\section{References}

1. Armitage JO, Weisenburger DD. New approach to classifying nonHodgkin's lymphomas: clinical features of the major histologic subtypes. Non-Hodgkin's lymphoma classification project. J Clin Oncol. 1998;16(8):2780-2795.

2. Nguyen T, Parker R, Hawkins E, et al. Synergistic interactions between PLK1 and HDAC inhibitors in non-Hodgkin's lymphoma cells occur in vitro and in vivo and proceed through multiple mechanisms. Oncotarget. 2017;8(19):31478-31493.

3. Laubach JP, San-Miguel JF, Hungria V, et al. Deacetylase inhibitors: an advance in myeloma therapy? Expert Rev Hematol. 2017;10(3): 229-237.

4. Lee YS, Lim KH, Guo X, et al. The cytoplasmic deacetylase HDAC6 is required for efficient oncogenic tumorigenesis. Cancer Res. 2008; 68(18):7561-7569.

5. Zhang Z, Yamashita H, Toyama T, et al. HDAC6 expression is correlated with better survival in breast cancer. Clin Cancer Res. 2004;10(20): 6962-6968.

6. Cai Y, Cui W, Chen W, et al. The effects of a histone deacetylase inhibitor on biological behavior of diffuse large B-cell lymphoma cell lines and insights into the underlying mechanisms. Cancer Cell Int. 2013;13(1):57.

7. Gao H, Gong Z. Beyond international prognostic index: risk stratification in diffuse large B-cell lymphoma. Transl Cancer Res. 2017;6: S210-S216.

8. Sterner DE, Berger SL. Acetylation of histones and transcription-related factors. Microbiol Mol Biol Rev. 2000;64(2):435-459.

9. Glozak MA, Seto E. Histone deacetylases and cancer. Oncogene. 2007;26(37):5420-5432.

10. Giannini G, Cabri W, Fattorusso C, Rodriquez M. Histone deacetylase inhibitors in the treatment of cancer: overview and perspectives. Future Med Chem. 2012;4(11):1439-1460.

11. Feng W, Zhang B, Cai D, Zou X. Therapeutic potential of histone deacetylase inhibitors in pancreatic cancer. Cancer Lett. 2014;347(2): 183-190.

12. Tasoulas J, Giaginis C, Patsouris E, Manolis E, Theocharis S. Histone deacetylase inhibitors in oral squamous cell carcinoma treatment. Expert Opin Investig Drugs. 2015;24(1):69-78.

13. Cheng T, Grasse L, Shah J, Chandra J. Panobinostat, a pan-histone deacetylase inhibitor: rationale for and application to treatment of multiple myeloma. Drugs Today. 2015;51(8):491-504. 
14. Grozinger CM, Hassig CA, Schreiber SL. Three proteins define a class of human histone deacetylases related to yeast Hda1p. Proc Natl Acad Sci U S A. 1999;96(9):4868-4873.

15. Verdel A, Khochbin S. Identification of a new family of higher eukaryotic histone deacetylases. Coordinate expression of differentiation-dependent chromatin modifiers. J Biol Chem. 1999;274(4):2440-2445.

16. Seidel C, Schnekenburger M, Dicato M, Diederich M. Histone deacetylase 6 in health and disease. Epigenomics. 2015;7(1):103-118.

17. Li Y, Shin D, Kwon SH. Histone deacetylase 6 plays a role as a distinct regulator of diverse cellular processes. FEBS J. 2013;280(3): 775-793.

18. Sakuma T, Uzawa K, Onda T, et al. Aberrant expression of histone deacetylase 6 in oral squamous cell carcinoma. Int J Oncol. 2006;29(1): $117-124$.

19. Medler TR, Craig JM, Fiorillo AA, Feeney YB, Harrell JC, Clevenger CV. HDAC6 deacetylates HMGN2 to regulate Stat5a activity and breast cancer growth. Mol Cancer Res. 2016;14(10):994-1008.

20. Ramakrishnan S, Ku S, Ciamporcero E, et al. HDAC 1 and 6 modulate cell invasion and migration in clear cell renal cell carcinoma. $B M C$ Cancer. 2016;16:617.

21. Hideshima T, Bradner JE, Wong J, et al. Small-molecule inhibition of proteasome and aggresome function induces synergistic antitumor activity in multiple myeloma. Proc Natl Acad Sci US A. 2005;102(24): 8567-8572.
22. Seo J, Min SK, Park HR, et al. Expression of histone deacetylases HDAC1, HDAC2, HDAC3, and HDAC6 in invasive ductal carcinomas of the breast. J Breast Cancer. 2014;17(4):323-331.

23. Batchu SN, Brijmohan AS, Advani A. The therapeutic hope for HDAC6 inhibitors in malignancy and chronic disease. Clin Sci. 2016;130(12): 987-1003.

24. Duvic M, Talpur R, Ni X, et al. Phase 2 trial of oral vorinostat (suberoylanilide hydroxamic acid, SAHA) for refractory cutaneous T-cell lymphoma (CTCL). Blood. 2007;109(1):31-39.

25. Olsen EA, Kim YH, Kuzel TM, et al. Phase IIb multicenter trial of vorinostat in patients with persistent, progressive, or treatment refractory cutaneous T-cell lymphoma. J Clin Oncol. 2007;25(21):3109-3115.

26. Marquard L, Gjerdrum LM, Christensen IJ, Jensen PB, Sehested M, Ralfkiaer E. Prognostic significance of the therapeutic targets histone deacetylase 1, 2, 6 and acetylated histone $\mathrm{H} 4$ in cutaneous T-cell lymphoma. Histopathology. 2008;53(3):267-277.

27. Marquard L, Poulsen CB, Gjerdrum LM, et al. Histone deacetylase 1, 2, 6 and acetylated histone $\mathrm{H} 4$ in B- and T-cell lymphomas. Histopathology. 2009;54(6):688-698.

28. Le GS, Milpied NJ, Lamy T, et al. First-line rituximab (R) high-dose therapy (R-HDT) versus R-CHOP14 for young adults with diffuse large B-cell lymphoma: preliminary results of the GOELAMS 075 prospective multicenter randomized trial. J Clin Oncol. 2011;29:8003.
OncoTargets and Therapy

\section{Publish your work in this journal}

OncoTargets and Therapy is an international, peer-reviewed, open access journal focusing on the pathological basis of all cancers, potential targets for therapy and treatment protocols employed to improve the management of cancer patients. The journal also focuses on the impact of management programs and new therapeutic agents and protocols on

\section{Dovepress}

patient perspectives such as quality of life, adherence and satisfaction. The manuscript management system is completely online and includes a very quick and fair peer-review system, which is all easy to use. Visit http://www.dovepress.com/testimonials.php to read real quotes from published authors. 